Classification

Physics Abstracts

82.70

\title{
Phase diagrams and shape transformations of toroidal vesicles
}

\author{
Frank Jülicher, Udo Seifert and Reinhard Lipowsky \\ Institut für Festkörperforschung, Forschungszentrum Jülich, 52425 Jülich, Germany \\ (Received 10 May 1993, accepted in final form 21 July 1993)
}

\begin{abstract}
Shapes of vesicles with toroidal topology are studied in the context of curvature models for the membrane. For two simplified curvature models, the spontaneous-curvature (SC) model and the bilayer-couple (BC) model, the structure of energy diagrams, sheets of stationary shapes and phase diagrams are obtained by solving shape equations for axisymmetric shapes. Three different sheets of axisymmetric shapes are investigated systematically: i) discoid tori; ii) sickle-shaped tori and iii) toroidal stomatocytes. A stability analysis of axisymmetric shapes with respect to symmetry breaking conformal transformations reveals that large regions of the phase diagrams of toroidal vesicles are non-axisymmetric. Non-axisymmetric shapes are determined approximately using conformal transformations. To compare the theory with experiments, a generalization of the $\mathrm{SC}$ and $\mathrm{BC}$ model, the area-difference-elasticity-model (ADE-model), which is a more realistic curvature model for lipid bilayers, is discussed. Shapes of toroidal vesicles which have been observed recently can be located in the phase diagram of the ADE-model. We predict the effect of temperature changes on the observed shapes. The new class of shapes, the toroidal stomatocytes, have not yet been observed.
\end{abstract}

\section{Introduction.}

Vesicles are closed surfaces, formed by lipid bilayers in aqueous solution [1-3]. The most general classification of such closed surfaces is by their topology. The different topological classes are distinguished by their genus, that is by the number of handles (or holes). Vesicles of spherical topology have been studied intensively, e.g. in experiments, where a change in temperature or osmotic conditions induces shape transformations between a variety of different shapes [4-6].

Recently, toroidal vesicles have been observed experimentally. The first examples were vesicles made of a partially polymerized bilayer [7]. Typically, these vesicles were close to one special axisymmetric circular shape, the so-called Clifford torus. Subsequently, toroidal vesicles consisting of fluid membranes have been found $[8,9]$. They exhibit a variety of different types of shapes of genus one, two and even higher genus. Among the genus- 1 surfaces observed so far, axisymmetric and non-axisymmetric shapes occur. The genus-2 surfaces which are 
necessarily non-axisymmetric can crudly be classified into two classes: i) discoid shapes with two holes and ii) shapes consisting of two spherical parts connected by three necks $[8,9]$.

Theoretical models for vesicles are based on the idea that the shape of a vesicle is determined by bending elasticity which can be expressed by the curvature of the surface. For spherical vesicles, the phase diagrams of two curvature models $[10,11]$, the spontaneous-curvature (SC) model and the bilayer-couple (BC) model have been studied systematically [12-14]. These phase diagrams are obtained by minimizing the bending energy for axisymmetric shapes with a given surface area and enclosed volume. Recently, a more realistic model, the area-differenceelasticity-model (ADE-model) has been proposed which takes into account that a bilayer is composed of two compressible monolayers which can glide over each other [15-18]. The SC and the $\mathrm{BC}$ model turn out to be mathematical limits of this more general model.

The systematic theoretical study of toroidal vesicles of genus-1 started with a minimal model for symmetric bilayers (without spontaneous curvature) with the volume to area ratio, i.e. the reduced volume, as the only parameter. Similar to the spherical topology, several branches of axisymmetric shapes were found and a one-dimensional phase diagram was determined [19, 20]. According to a famous conjecture the axisymmetric shape of lowest energy irrespective of the reduced volume, the Clifford torus, is an absolute energy minimum [21]. Since the bending energy is a conformal invariant, this ground state is degenerate, i.e. a one-parameter family of non-axisymmetric shapes with the same energy can be generated from the Clifford torus by applying conformal transformations [22]. The physical constraint of a fixed volume to area ratio breaks this degeneracy. Consequently, the ground state at fixed reduced volume is unique but non-axisymmetric over a large range in the reduced volume $[20,23]$. In the vicinity of the Clifford torus, the eigenmodes of the bending energy which lead to axisymmetry breaking have been calculated analytically $[24,25]$.

In this paper, we aim for a thorough theoretical analysis of toroidal vesicle shapes from which statements about recent experimental observations as well as predictions for future experiments can be infered. We start in section 2 by analysing the two curvature models, the $\mathrm{SC}$ and the $\mathrm{BC}$ model, which both have been studied intensively for vesicles of spherical topology. We classify the sheets [26] of stationary shapes and determine the phase diagrams for both models. Even though, as outlined in section 3, there are good reasons to believe that neither of the two models gives a faithful description of the bilayer membrane, a detailed study of these (from our present perspective) "simplified" or "mathematical" curvature models, is justified. First of all, important insight into the structure of the possible shapes can already be obtained in these models. Secondly, the phase diagram for a more realistic model, the area-difference-elasticity (ADE) model as discussed in section 3.1, can easily be derived by a general Legendre transformation from the phase diagram of the $\mathrm{BC}$ model. The phase diagram of the ADE-model, then provides the basis for a comparison with recent experiments in section 3.2. Moreover, we can make predictions for future experiments in section 3.3.

To facilitate a coherent reading of the paper, we give in section 2 only results while relegating all important technicalities to the appendices. The shape equations for toroidal topology are derived in appendix $A$. In appendix B, we discuss in detail the energy diagrams for stationary shapes in the SC and the BC model as well as the different types of limit shapes. In appendix $\mathrm{C}$, the stability criterion of axisymmetric shapes with respect to symmetry breaking conformal transformations is derived. 


\section{Simplified curvature models.}

2.1 Spontaneous-curvature model. - In the SC model of Helfrich, the energy of the bilayer is given by [10]

$$
F \equiv \frac{\kappa}{2} \oint \mathrm{d} A\left(C_{1}+C_{2}-C_{0}\right)^{2}+\kappa_{\mathrm{g}} \oint \mathrm{d} A C_{1} C_{2}
$$

Here, $C_{1}$ and $C_{2}$ denote the principal curvatures on the surface, while $\kappa$ and $\kappa_{g}$ are the ordinary and the Gaussian bending rigidity. The spontaneous curvature $C_{0}$ is a model parameter that arises if the symmetry between the two monolayers is broken. Physical examples for this case are: i) a membrane with two different monolayers forming a bilayer or ii) a bilayer facing two different solvents. The Gauß-Bonnet theorem states that the second term is invariant under transformations which do not change the topology of the surface. As long as the topology is fixed, this term can and will be obmitted.

The phase diagram is determined by minimizing the energy $F$ for fixed area $A$ of the surface and fixed enclosed volume $V$. Since $F$ is invariant under scale-transformations, there are only two independent parameters. These are the reduced volume

$$
v \equiv \frac{V}{4 \pi R_{0}^{3} / 3}
$$

and the reduced spontaneous curvature

$$
c_{0} \equiv C_{0} R_{0}
$$

where

$$
R_{0} \equiv(A / 4 \pi)^{1 / 2}
$$

is the radius of a sphere with surface area $A$. Then, the energy $F=F\left(v, c_{0}\right)$ and the phase diagram depend on $v$ and $c_{0}$ only.

2.2 Bilayer-COUPLE MOdel. - In the BC model [14] the bending energy reads

$$
G \equiv \frac{\kappa}{2} \oint \mathrm{d} A\left(C_{1}+C_{2}\right)^{2}
$$

As a third constraint, the area difference

$$
\Delta A \equiv A^{\mathrm{ex}}-A^{\mathrm{in}} \approx 2 D M
$$

between the outer and the inner monolayer is kept fixed, which can be calculated from the total mean curvature

$$
M \equiv \frac{1}{2} \oint \mathrm{d} A\left(C_{1}+C_{2}\right)
$$

and the distance $D$ between the neutral surfaces of the monolayers. Physically, the constraint on $\Delta A$ would describe incompressible monolayers which do not exchange lipid molecules. In this case the energy $G=G(v, m)$ can be expressed by the reduced volume $v$ and the reduced total mean curvature

$$
m \equiv M / R_{0}
$$

As derived in reference [12], the stationary shapes are the same in both models. The phase diagrams are, however, completely different. 
2.3 STATIONARY SHAPES OF AXIAL SYMMETRY: SHEETS OF SOLUTIONS. - Stationary shapes of axial symmetry are determined by solving the shape equations (A4-A9) derived in appendix A. The solutions to these coupled nonlinear differential equations are a discrete set of sheets with different energies $F$ or $G$. Stationary shapes forming such a sheet can be either saddle points or local minima, i.e. equilibrium shapes, of the curvature energy. The distinction between both cases requires a complete stability analysis. However, stability with respect to deformations preserving axisymmetry can be checked by close inspection of the energy diagrams as discussed in appendix B. In order to study stability with respect to non-axisymmetric deformations, we use an approximation based on conformal transformations as described in the next section.

Three different sheets of stationary shapes can be distinguished, see figure 1: i) the sheet of sickle-shaped tori; ii) the sheet of discoide tori and iii) the sheet of toroidal stomatocytes which do not have a symmetry plane perpendicular to the symmetry axis.

Both, the discoide tori and the sickle-shaped tori have reflection symmetry with respect to the plane perpendicular to the axis of symmetry. They can be distinguished as different sheets since they are separated from each other except for one shape which is the Clifford torus. Therefore, starting with the Clifford torus which has an exactly circular cross section, the two different ellipsoidal deformations of the contour lead to the sheets of discoide and sickle-shaped tori [27]. The sheet of toroidal stomatocytes bifurcates from the sheet of sickle-shaped tori and from the sheet of discoide tori, thus connecting these two sheets. As an illustrative example, we show in figure 1a the energy $G$ of the sheets of stationary shapes versus the reduced total mean curvature $m$ for shapes with fixed reduced volume $v=0.55$. The detailed discussion of this diagram is given in appendix $\mathrm{B}$. In figure $1 \mathrm{~b}$, we show the corresponding calculated contours of shapes of lowest energy $G$.

Various types of limit shapes, where the sheets end and the shape becomes singular, can be distinguished. For all three sheets, one class of limit shapes occurs, where the hole diameter vanishes. Formally, these shapes represent a connection to the spherical topology and are denoted by $\mathrm{L}_{\text {sick }}, \mathrm{L}_{\text {disc }}$ and $\mathrm{L}_{\text {sto }}$. Other classes of limit shapes include tori with exactly circular cross section $\mathrm{L}_{\text {circ }}$ and shapes with diverging hole diameter. A detailed discussion of the limit shapes is given in appendix $B$.

2.4 AXIAL-SYMMETRY-BREAKING CONFORMAL TRANSFORMATIONS. - The stationary shapes with axial symmetry can be obtained by solving shape equations for the contour. Nonaxisymmetric shapes become accessible through conformal transformations applied to axisymmetric shapes. The usefulness of this approximate method to determine the full phase diagram is first shown using the Clifford torus.

2.4.1 The Clifford torus and its conformal transformations. - The Clifford torus is an axisymmetric shape with a circular cross section and a fixed ratio of $\sqrt{2}$ of its generating radii with reduced volume $v=v_{\mathrm{cl}} \equiv 3 /\left(2^{5 / 4} \pi^{1 / 2}\right) \simeq 0.71$ and the reduced total mean curvature $m=m_{\mathrm{cl}} \equiv \pi^{3 / 2} 2^{5 / 4} \simeq 13.24$. The Clifford torus is a stationary shape of the energy $F$ for any value of $C_{0}$ [28].

An important property of this shape was conjectured by Willmore [21]: for genus-1 surfaces, the Clifford torus is the absolute minimum of the bending energy

$$
G \equiv \frac{\kappa}{2} \oint \mathrm{d} A\left(C_{1}+C_{2}\right)^{2}
$$

with $G=G_{\mathrm{cl}} \equiv 4 \pi^{2} \kappa$. 

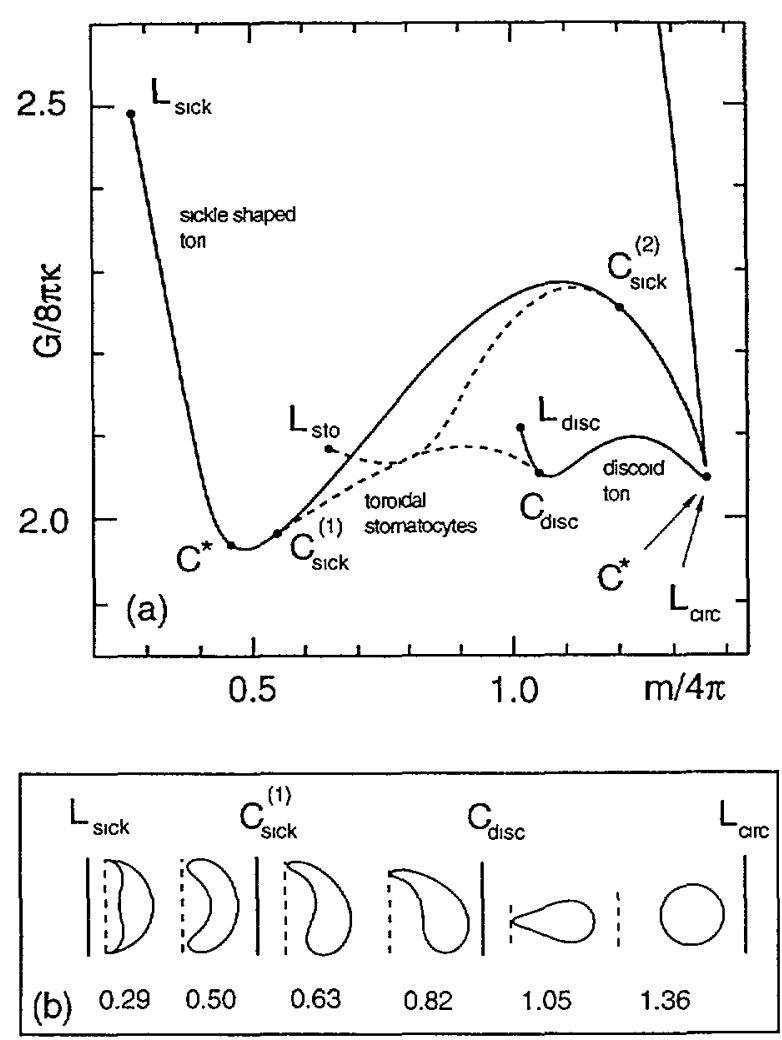

Fig.1. - a) Energy $G$ of the axisymmetric stationary shapes in the BC model versus the total mean curvature $m$ for fixed reduced volume $v=0.55$. Three different branches can be distinguished: i) a branch of discoid tori; ii) a branch of sickle-shaped tori and iii) a branch of toroidal stomatocytes. The toroidal stomatocytes bifurcate from the other branches at the points $C_{\text {sick }}^{(1)}, C_{\text {sick }}^{(2)}$ and $C_{\text {disc }}$. All three branches end at different types of limit shapes: $L_{\text {sick }}, L_{s t o}$ and $L_{\text {disc }}$ are limit shapes with a vanishing hole diameter. At $\mathrm{L}_{\text {circ, }}$ a limit shape with perfectly circular cross section is reached. Some parts of these axisymmetric branches are unstable with respect to symmetry breaking conformal transformations. These instabilities occur at the points $C^{*}$. b) Sequence of axisymmetric shapes of minimal energy $G$ for fixed reduced volume $v=0.55$ and several values of $m / 4 \pi$. The first and the last shape are unstable with respect to non-axisymmetric deformations.

Since the bending energy $G$ is an invariant under conformal transformations in the threedimensional embedding space, every shape that is a conformal transformation of the Clifford torus also has the same energy $G$. Consequently, the energy minimum for tori is degenerate as first observed by Duplantier [22] if no further constraints are imposed.

The nontrivial conformal transformations in three dimensions are the special conformal transformations which can be parametrized by a vector $a$. This transformation consists of an inversion $\mathbf{R} \rightarrow \mathbf{R} / R^{2}$, a translation by a vector a and another inversion. Every point $\mathbf{R}$ is thus transformed to $\mathbf{R}^{\prime}$, with

$$
\mathbf{R}^{\prime}=\frac{\mathbf{R} / R^{2}+\mathbf{a}}{\left(\mathbf{R} / R^{2}+\mathbf{a}\right)^{2}}
$$

Note, that two successive special conformal transformations (10) with translation vectors $\mathbf{a}_{1}$ 

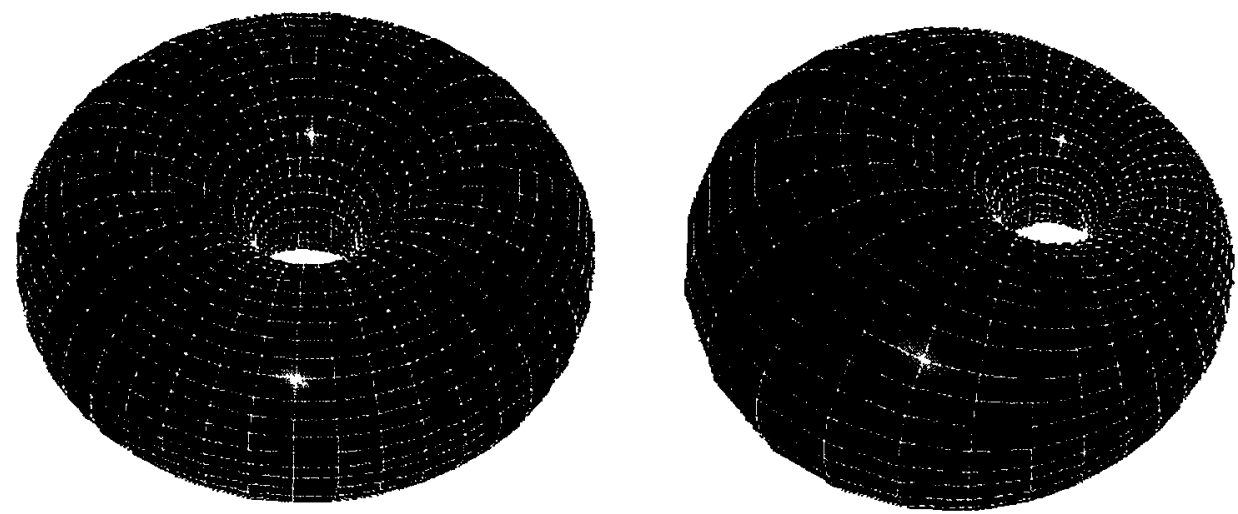

Fig.2. - The Clifford torus and a shape generated by a special conformal transformation as in (10) with translation vector $a_{X}=0.3 / R_{0}$, and $a_{Y}=a_{Z}=0$.

and $\mathbf{a}_{2}$ are equivalent to one special conformal transformation with $\mathbf{a}=\mathbf{a}_{1}+\mathbf{a}_{2}$, i.e., the special conformal transformations form a commutative subgroup.

Conformal transformations of the Clifford torus generate a one-parameter family of nonaxisymmetric shapes. This can be seen by applying a conformal transformation (10) to the Clifford torus with the $Z$-axis as the axis of rotational symmetry and the $X-Y$-plane as the symmetry plane, see figure 2 as an example. First, choose a vector $\mathbf{a}=(\lambda \cos \phi, \lambda \sin \phi, 0)$. Varying its length $\lambda$ generates a one-parameter family of non-axisymmetric shapes with varying reduced volume $v_{\mathrm{cl}} \leq v(\lambda)<1$. This family of shapes can be described in a closed analytical form [25]. For $\lambda=2^{1 / 4} \pi^{1 / 2} /\left(R_{0}\left(2^{1 / 2}+1\right)\right)$, it ends up at a limit shape with $v=1$. This limit shape consists of a sphere with an infinitesimal handle. All these shapes have the same bending energy $G_{\mathrm{cl}}=4 \pi^{2} \kappa$. Therefore, the handle gives a finite contribution $4 \pi^{2} \kappa-8 \pi \kappa$ to the bending energy, since $G=8 \pi \kappa$ for a sphere. On the other hand, if a special conformal transformation with $\mathbf{a}=\left(0,0, a_{Z}\right)$ is applied to the Clifford torus, this leads only to scale-transformations and no new shapes are generated. The latter property depends crucially on the circular cross section of the Clifford torus. In contrast, for a general toroidal shape with $X-Y$ symmetry plane (e.g. sickle-shaped tori), a conformal transformation $(10)$ with $\mathbf{a}=\left(0,0, a_{Z}\right)$ does generate a shape with broken up-down symmetry. Thus, even though the special conformal transformations involve the three parameters $\left(a_{X}, a_{Y}, a_{Z}\right)$, there is only a one-parameter family of special conformal transformations which produces new shapes when applied to the Clifford torus due to its special symmetry properties. Therefore, for fixed $v$, there is no conformal degeneracy that would lead to conformal modes (or "massless Goldstone modes") [23].

2.4.2 Axial symmetry-breaking: approximate phase boundaries. - The non-axisymmetric toroidal shapes of lowest bending energy, as obtained via conformal transformations of the Clifford torus, represent the ground-state: i) for $c_{0}=0$ and $v>v_{\mathrm{cl}}$ in the SC model and ii) along a line $v=v_{c}(m)$ in the $\mathrm{BC}$ model. This indicates, that regions of non-axisymmetric shapes exist in the phase diagrams of both models [29].

We now apply the hypothesis that conformal transformations of the various sheets of axisymmetric shapes provide a reasonable estimate for the variational solution for all regions of the phase diagram. Specifically, we analyse the infinitesimal stability of the axisymmetric sheets with respect to special conformal transformations which can be calculated from the contour line of this shape [30]. This method gives a lower bound on that region of the phase diagram 

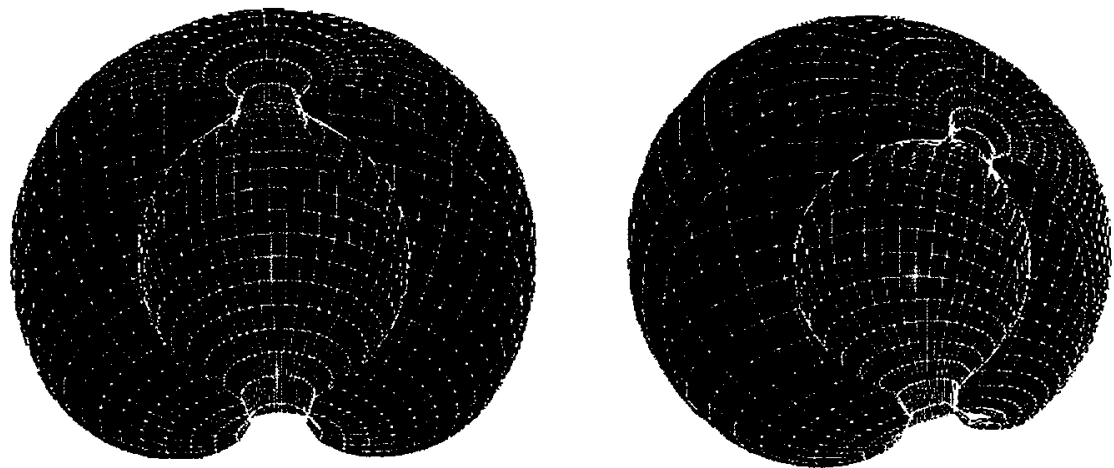

Fig.3. - An axisymmetric sickle-shaped torus with $v=0.55$ and $m / 4 \pi=0.5$ and a nonaxisymmetric sickle-shaped torus. The non-axisymmetric shape was generated by applying the special conformal transformation (10) with $a_{X}=0.3 / R_{0}$, and $a_{Y}=a_{Z}=0$ to the axisymmetric shape.

in which the ground state is non-axisymmetric. The idea behind this method is to start with a shape $\mathcal{S}_{1}$ on the sheet of lowest energy shapes and apply a special conformal transformation to $\mathcal{S}_{1}$ with $a_{X} \neq 0$, see figure 3 for an example. This transformation generates a new nonaxisymmetric shape, $\mathcal{S}_{1}^{\prime \prime}$, with the same bending energy as $\mathcal{S}_{1}$ which is located on a different point in the phase diagram. The stability of the original shape now depends on the difference of the energy of $\mathcal{S}_{1}^{\prime \prime}$ and the energy of the axisymmetric shape $\mathcal{S}^{\text {axi }}$ which is located at the same point in the phase diagram. The complete derivation of this stability criterion is given in appendix $\mathrm{C}$.

2.5 Phase diagram of The SPontaneous-Curvature Model. - The analysis of the stationary axisymmetric shapes together with the stability analysis with respect to conformal transformations leads to phase diagrams. Quite generally, a phase diagram consists of several regions where the shapes have different symmetry properties. Transformations between shapes of different regions are called shape transitions.

The phase diagram for the SC model is shown in figure 4. It contains three regions: i) a region of axisymmetric sickle-shaped tori; ii) a region of tori with nearly circular cross section ("circular tori") [31] and iii) a large region of non-axisymmetric shapes. The two axisymmetric regions are separated by the discontinuous phase boundary $\mathrm{D}_{\mathrm{ax}}$. The axisymmetry of the sickle shaped tori and the circular tori is broken continuously at the lines $\mathrm{C}_{\text {sick }}^{*}$ and $\mathrm{C}_{\text {disc }}^{*}$, respectively. These lines end up at two different critical endpoints $\mathrm{D}_{1}$ and $\mathrm{D}_{2}$ on the discontinuous transition line $D_{a x}$. The line $D_{a x}$ extends into the non-axisymmetric region until it ends up in the critical point $\mathrm{D}_{\mathrm{cp}}$. The Clifford torus is located along a line CL with $v=v_{\mathrm{cl}}$ which connects the lines $\mathrm{C}_{\text {disc }}^{*}$ and $\mathrm{D}_{\mathrm{ax}}$.

The lines $\mathrm{C}_{\text {sick }}^{*}$ and $\mathrm{C}_{\text {disc }}^{*}$ give the instabilities with respect to axisymmetry breaking if deformations are restricted to conformal transformations. Thus, they represent bounds for the instabilities which would have been obtained by a full stability analysis of the axisymmetric sheets: the non-axisymmetric regions extend at least to the lines $\mathrm{C}_{\text {sick }}^{*}$ and $\mathrm{C}_{\text {disc }}^{*}$. For the Clifford torus, the conformal mode is the unstable eigenmode. To get an idea of the shapes in the non-axisymmetric region close to $\mathrm{C}_{\text {disc }}^{*}$, a conformally unstable axisymmetric stationary shape can be used. By applying a conformal transformation to such a shape, non-axisymmetric shapes are generated which should be good approximations for shapes of minimal energy in 


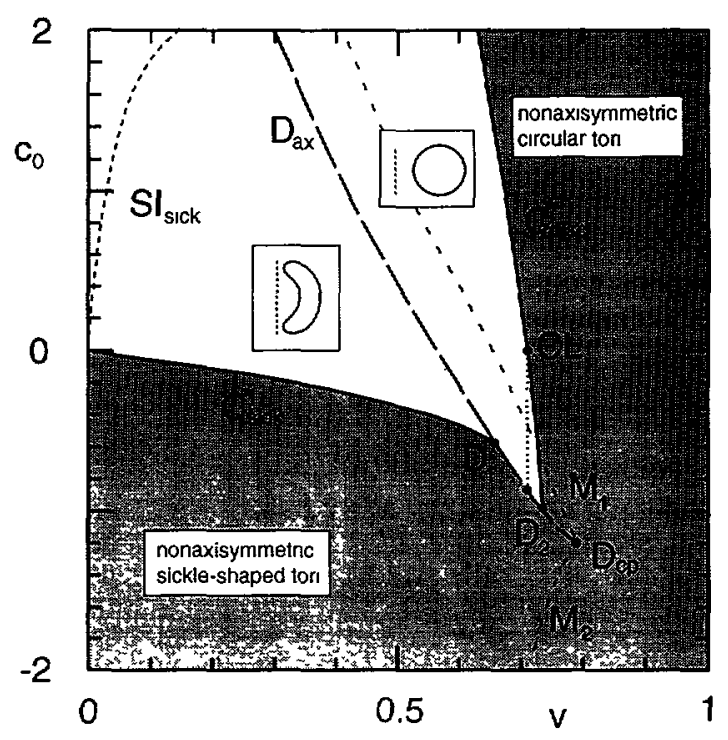

Fig.4. - Phase diagram for toroidal vesicles in the SC model. The two model parameters are the reduced volume $v$ and the reduced spontaneous curvature $c_{D}$. The line $\mathrm{D}_{\mathrm{ax}}$ which ends in the critical point $D_{c p}$, represents the line of discontinuous shape transformations. It separates a region of circular tori from a region of sickle-shaped tori. At the dotted line $\mathrm{SI}_{\text {sick, }}$, the sickle-shaped tori selfintersect. The two dashed lines $\mathrm{C}_{\text {sick }}^{*}$ and $\mathrm{C}_{\mathrm{disc}}^{*}$ represent lines of instabiliy of axisymmetric shapes with respect to symmetry breaking conformal transformations. They serve as approximations for the exact continuous shape transformations of axisymmetry breaking. Two different types of non-axisymmetric shapes can be distinguished: i) non-axisymmetric sickle-shaped tori and ii) non-axisymmetric circular tori. For $c_{0}=0$, the Clifford torus (CL) appears in the phase diagram on the line $\mathrm{C}_{\mathrm{d} 1 s c}^{*}$. The Clifford torus is the minimal energy shape along the dotted line with $v=v_{\mathrm{cl}}$.

this region. An example for a shape generated in this way is the conformally transformed Clifford torus shown in figure 2. Likewise, a conformally unstable axisymmetric sickle-shaped torus can be used to approximate non-axisymmetric sickle-shaped tori below $\mathrm{C}_{\text {sick}}^{*}$, see figure 3.

The lines $M_{1}$ and $M_{2}$ represent borders of metastability related to the discontinuous transitions along the line $D_{\mathrm{ax}}$. Starting, for example, from the region of sickle-shaped tori, crossing the line $\mathrm{D}_{\mathrm{ax}}$, the sickle-shaped tori become metastable while the circular tori are shapes of minimal energy. At $M_{1}$, the metastable sickle-shaped tori become unstable. Similarly, metastable circular tori become unstable at $\mathrm{M}_{2}$. Along the line $\mathrm{SI}_{\text {sick }}$ the contour of the sickle-shaped tori selfintersects in the symmetry plane of these shapes. For a calculation of shapes beyond this line, one would have to include selfinteractions of the vesicle membrane.

2.6 Phase diagram of the bilayer-Couple model. - Figure 5 shows the phase diagram of the $\mathrm{BC}$ model which depends on the reduced volume $v$ and the reduced total mean curvature $m$. For a closed surface, the surface average of the mean curvature is positive and therefore $m>0$. Four different regions can be distinguished: i) a region of discoid tori; ii) a region of sickle-shaped tori; iii) a region of toroidal stomatocytes and iv) a large region of nonaxisymmetric tori. 


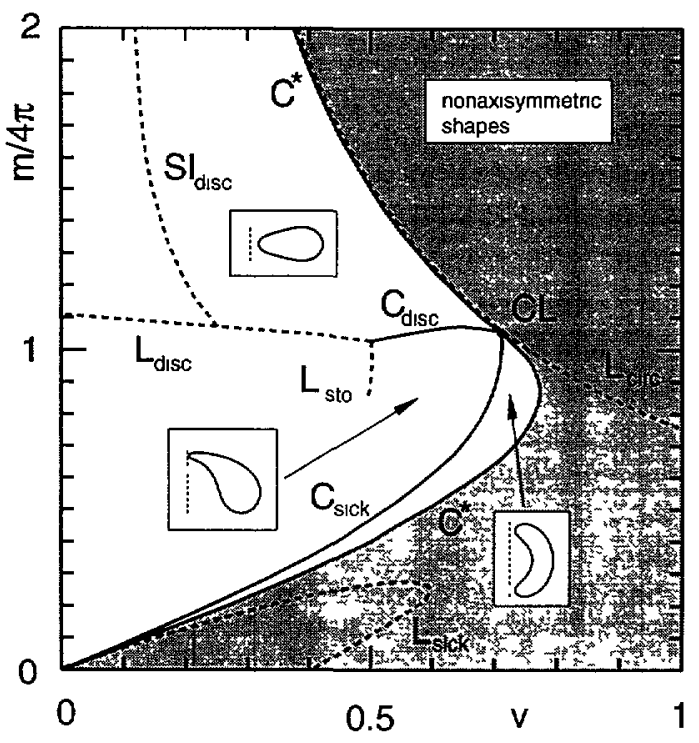

Fig.5. - Phase diagram for toroidal vesicles in the $\mathrm{BC}$ model. Three axisymmetric regions which are separated by continuous shape transformation lines $\mathrm{C}_{\text {sick }}$ and $\mathrm{C}_{\text {disc }}$ can be distinguished: i) discoid tori; ii) sickle-shaped tori and iii) toroidal stomatocytes. The line $\mathrm{C}^{*}$ indicates the instability with respect to axisymmetry breaking conformal transformations. On the right hand side of this line, nonaxisymmetric shapes have minimal energy. The lines $L_{\text {disc }}, L_{\text {sto }}$ and $L_{\text {sick }}$ represent limit shapes with vanishing hole diameter. Above the line of circular limit shapes $L_{c i r c}$, no axisymmetric stationary shapes exist. The contour of discoid tori begins to selfintersect at the line $\mathrm{SI}_{\text {disc. }}$.

The mirror symmetry planes of the discoid tori and of the sickle-shaped tori are broken continuously at the lines $\mathrm{C}_{\text {disc }}$ and $\mathrm{C}_{\text {sick }}$, respectively, leading to the region of toroidal stomatocytes without a reflection symmetry plane.

Continuous axisymmetry breaking occurs along the line $\mathrm{C}^{*}$ which separates a region of nonaxisymmetric shapes for relatively large $v$ in the phase diagram from the region of axisymmetric shapes for relatively small $v$ [32]. This line begins at large $m$ with discoid tori with a nearly circular cross section. It continues with sickle-shaped tori until it ends in the point $(v, m)=$ $(0,0)$. A special shape on this line is the Clifford torus, again denoted by CL. In this phase diagram, the Clifford torus is a multicritical point where the continuous shape transformation lines $\mathrm{C}_{\text {disc }}, \mathrm{C}_{\text {sick }}$ and $\mathrm{C}^{*}$ meet.

The line $\mathrm{C}^{*}$ was obtained by analyzing the conformal instability of axisymmetric shapes. This conformal instability which occurs in the $\mathrm{BC}$ model for the same shapes as in the SC model, is again a lower bound for the extension of the region of non-axisymmetric shapes. It becomes the correct phase boundary at the Clifford torus CL. The examples of non-axisymmetric shapes as shown in figures 2 and 3 hold for the $\mathrm{BC}$ model too.

The dotted lines in the phase diagram represent lines of limit shapes and selfintersection, as described in appendix B. A general property of the phase diagram of the $\mathrm{BC}$ model is, that all shape transformations are continuous as it has previously been observed for spherical shapes [12]. However, we are not aware of any reason for the absence of first order transitions in the $\mathrm{BC}$ model for all topologies. 


\section{Relation to experiments: The ADE-model.}

3.1 THE ADE-MODEL. - The two curvature models described so far seem to contain essential features of the bilayer membrane. Reflection on the physical assumptions underlying these models, however, reveals that both of them give only a crude representation of one important aspect of the physical properties of the bilayer system. The two monolayers forming a fluid lipid bilayer are able to slide with respect to each other with relative ease. When such a bilayer forms a closed vesicle and experiences changes in local curvatures, there will be a net expansion or compression of the surface area of the individual monolayers of the order $D / R_{0}$. Since relative movement can take place between the leaves, such expansion or compression will be homogeneous over the whole surface or "non-local" rather than location dependent [11]. Neither the SC model nor the BC model has incorporated this effect. The SC model neglects this non-local factor, since it implicitly assumes that the two monolayers are rigidly connected and have no relative movement, thus being essentially a model for interdigitated monolayers. On the other hand, the $\mathrm{BC}$ model recognizes the specific way of coupling between the two monolayers but does not allow any deviation of the monolayer surface area from its equilibrium value, assuming a zero area-compressibility for the individual monolayers.

We will now consider a model where this non-local bending energy is incorporated. In addition, it will be assumed that the number of lipid molecules remains constant within each monolayer, i.e., that no exchange of lipid molecules occurs between the two adjacent monolayers even though their density is now different. This is the area-difference-elasticity (ADE) model. Its energy reads in scaled variables $[15,16,18]$

$$
W \equiv \frac{\kappa}{2}\left[\oint\left(C_{1}+C_{2}\right)^{2} \mathrm{~d} A+\alpha\left(m-m_{0}\right)^{2}\right]=G+\frac{\kappa \alpha}{2}\left(m-m_{0}\right)^{2}
$$

The second term explicitly attributes an elastic energy to deviations of the area difference $\Delta A \equiv 2 D R_{0} m$ from an equilibrium value $\Delta A_{0} \equiv 2 D R_{0} m_{0}$. The area difference $\Delta A_{0}$ of the relaxed bilayer depends on the preparation of each individual vesicle. The dimensionless parameter $\alpha$ is the ratio of area difference elastic energies to the usual bending energies and is for realistic bilayers close to one. For the calculation of the phase diagram, we choose $\alpha=1$ which is supported by the so far only measurement of this quantity [18]. In the limits of large $\alpha$ and small $\alpha$, the $\mathrm{BC}$ model and the SC model are recovered, respectively.

The phase diagram of the ADE-model can be derived from that of the $\mathrm{BC}$ model as follows: the energy $W$ has to be minimized for fixed enclosed volume $V$, surface area $A$ and $m_{0}$. Stationary shapes of the energy $W$ of the ADE-model can be determined by first varying $W$ with respect to the subspace of shapes with fixed reduced total mean curvature $m$. This leads to the energy

$$
W\left(v, m, m_{0}\right)=G(v, m)+\kappa \alpha\left(m-m_{0}\right)^{2} / 2
$$

The final variation of $W\left(v, m, m_{0}\right)$ with respect to $m$ for fixed $m_{0}$ leads to

$$
\frac{\partial G(m, v)}{\partial m}=-\kappa \alpha\left(m-m_{0}\right)
$$

This relation determines the value of $m$ as a function of $m_{0}$. Inserting this value of $m$ in equation (12) leads to the energy $W\left(m_{0}, v\right)$ of stationary shapes of the ADE-model. The curves of conformal instability in the $\mathrm{BC}$ model can be transformed by the same generalized Legendre transformation to the ADE-model since the conformal stability of axisymmetric shapes with mirror symmetry plane does not depend on $\alpha$ [33].

In figure 6 , we show the phase diagram of toroidal vesicles in the ADE-model. It depends on two parameters, the reduced volume $v$ and $m_{0}$. This phase diagram is qualitatively sim- 


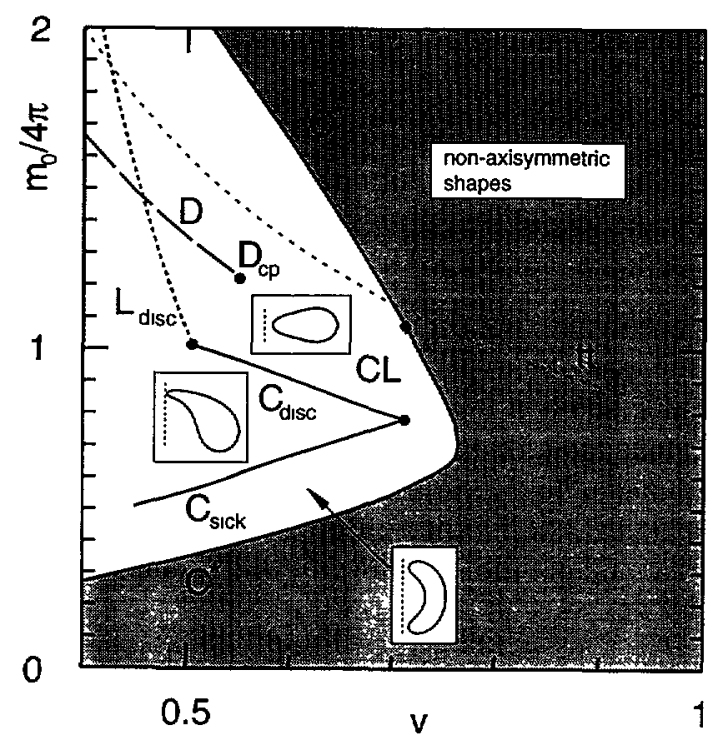

Fig.6. - Phase diagram for toroidal vesicles in the ADE-model with $\alpha=1$. The phase diagram is very similar to that of the $\mathrm{BC}$ model explained in figure 5 . Three axisymmetric regions which are separated by continuous shape transformation lines $\mathrm{C}_{\text {sick }}$ and $\mathrm{C}_{\text {disc }}$ can be distinguished: i) discoid tori; ii) sickle-shaped tori and iii) toroidal stomatocytes. The line $L_{\text {disc }}$ represent limit shapes with vanishing hole diameter. The instability with respect to axisymmetry breaking conformal transformations is denoted by $\mathrm{C}^{*}$. Within the region of axisymmetric shapes with reflection plane, discontinuous shape transformations from circular tori to discoid tori occur along the line $D$. This line ends up in a critical point $D_{c p}$. The Clifford torus is the shape of minimal energy along the dotted line CL. A temperature change corresponds to a temperature trajectory as indicated by the dashed line ( $t \mathrm{t})$. This trajectory crosses the line $\mathrm{C}^{*}$ which implies that axisymmetry breaking shape transformations of toroidal vesicles can be induced by temperature changes.

ilar to the phase diagram of the $\mathrm{BC}$ model, compare figure 5. There is a large region of non-axisymmetric shapes which are separated from the axisymmetric shapes by the phase boundary $\mathrm{C}^{*}$. All sheets of axisymmetric shapes appear within the ADE-model as shapes of minimal energy: i) discoid tori; ii) sickle-shaped tori and iii) toroidal stomatocytes. Symmetry breaking shape transformations occur at the lines $\mathrm{C}^{*}, \mathrm{C}_{\text {disc }}$ and $\mathrm{C}_{\text {sick}}$. These transitions are all continuous. A discontinuous transition line $D$ which ends in the critical point $D_{c p}$ separates discoid tori from tori with a circular cross section within the region of mirror symmetric axisymmetric shapes. While in the $\mathrm{BC}$ model the Clifford torus is a multicritical point where all symmetry breaking phase boundaries meet, a whole line CL of Clifford tori exists in the phase diagram of the ADE-model, as it does in the SC model.

3.2 EXPERIMENTALLY OBSERVED SHAPES: LOCATION IN THE PHASE DIAGRAM. - The observations by Fourcade, Mutz and Bensimon include shapes of genus one and genus two. Vesicles of genus one can be immediately located in the phase diagram for toroidal vesicles in the ADE-model. As a first example, consider the axisymmetric circular torus shown in reference [8] for which the authors estimate $v \simeq 0.5$. The value of $m_{0}$ cannot be measured directly. In principle, it could be obtained by carefully comparing the contour of the observed torus with 
the solutions of the shape equations. Inspection of the phase diagram shows, that circular shapes with $v=0.5$ exist within a large range of $m_{0}$, indeed. Second, consider the observed non-axisymmetric torus with $v \simeq 0.77$. This shape resembles the non-axisymmetric shape shown in figure 2. The phase diagram of the ADE-model shows that shapes with $v \gtrsim 0.77$ are definitely non-axisymmetric for all $m_{0}$.

Let us now discuss the genus-2 shapes seen by Fourcade et al.. Even without an explicit calculation of minimal energy shapes with $g>1$, which are necessarily non-axisymmetric, the observed genus- 2 shapes can be related to calculated genus- 1 shapes which occur in the phase diagram. This can be seen by starting e.g. with a discoid torus in the limit where the hole diameter vanishes (i.e. with a limit shape $L_{\text {disc }}$ as discussed in appendix $B$ ). In this case, the hole gives no contribution to the bending energy and the shape is identical to a spherical discocyte. If now a second infinitesimal hole is inserted, the energy will only change infinitesimally. This indicates that mirror-symmetric discoid shapes, with two holes in this symmetry plane, occur in the phase diagram of genus-2 vesicles [34]. They are related to discoid tori of genus one. An example of such a discoid shape of genus 2 has been reported in reference [8].

By a similar argument sickle-shaped tori can be related to the second type of genus- 2 shapes observed so far. In the limit of zero $v$, the sickle-shaped tori consist of two concentric spheres of equal radii connected by two infinitesimal necks. Inserting a third infinitesimal neck to a sickle-shaped torus with infinitesimal $v$ does not change the energy. This indicates that shapes consisting of two spherical parts connected by three necks are genus- 2 shapes with minimal energy for small $v$ and small $m$. A third neck breaks the axisymmetry of the original sickleshaped tori, but can be arranged in such a way that the positions of the necks obey a threefold symmetry. Conformal transformations applied to such a shape will induce a shift of the neck positions comparable to the one shown in figure 3 for the genus- 1 torus. In fact, a shape with fluctuating neck positions has recently been observed [9].

3.3 Predictions FOR FUTURE EXPERIMEnts. - So far, in the experiments by Bensimon and co-workers shapes with non-trivial topology are recorded without changing external parameters. For a crucial test of the theory presented here, one should vary one parameter systematically. Temperature changes have successfully been used for vesicles of spherical topology to induce shape transitions $[4,5]$. We recall that a change in temperature affects both $v$ and $m_{0}$ because of the thermal expansivities of the monolayers of the membrane and the enclosed water. These temperature trajectories in the phase diagram, and the corresponding sequence of shape transformations, are significantly affected by any small asymmetry of the order of $10^{-3}$ in the thermal expansion coefficients of the two monolayers [4, 12]. So far, there are no independent measurements or estimates for such a possible asymmetry. In this paper, we therefore discuss the ideal case of symmetric thermal expansion of the monolayers. If the total bilayer volume is constant, temperature trajectories can be described by [12]

$$
m_{0}=\left(\bar{m}_{0} \bar{v}\right) / v
$$

where $\left(\bar{v}, \bar{m}_{0}\right)$ denotes a starting point in the phase diagram. Figure 6 shows such a temperature trajectory $(t t)$. For decreasing temperature the reduced volume $v$ increases while $m_{0}$ decreases. Starting in the axisymmetric region of discoid tori, the trajectory crosses the continuous shape transformation $\mathrm{C}^{*}$ where the axisymmetry is broken. Thus, the model predicts that for any circular torus a decrease in temperature will lead to a non-axisymmetric torus via a continuous shape transformation (provided the bilayer remains in the fluid state). Since this transformation is reversible, increasing the temperature, i.e. reducing $v$, renders 
the vesicle axisymmetric again. This prediction can be extended to all axisymmetric shapes which include the sickle-shaped tori and the toroidal stomatocytes described below: starting with any axisymmetric shape, a decrease in temperature leads to a non-axisymmetric shape via a continuous shape transition which breaks the axisymmetry. In the phase diagram of the ADE-model with $\alpha=1$, metastable shapes exist only within a very small stripe along the discontinuous line $\mathrm{D}$. Therefore, almost all shape changes are reversible in this case. This differs significantly from the situation in the $\mathrm{SC}$ model, which exhibits a large region between the lines $M_{1}$ and $M_{2}$, where the vesicle would stay in a metastable state because of the large activation barrier for the first order transition.

Finally, a new type of toroidal shapes which has not been observed yet is predicted by our phase diagram: two continuous shape transformations, $C_{\text {disc }}$ and $C_{\text {sick }}$, separate the toroidal stomatocytes without reflection plane from the shapes with reflection plane. At these phase boundaries, the mirror symmetry is broken continuously. The phase diagram shows that the region of toroidal stomatocytes can not be reached via a temperature trajectory from the circular or non-axisymmetric shapes described so far. However, if it was possible to decrease $m_{0}$ at fixed $v$, circular torus should loose its reflection plane and become a toroidal stomatocyte. For even smaller $m_{0}$, the reflection symmetry is restored at $\mathrm{C}_{\text {sick }}$. The area difference $m_{0}$ of the monolayers can be changed without changing the reduced volume by redistributing the lipids through the bilayer. For vesicles of spherical topology, such a redistribution has been induced by Farge and Devaux with a mixture of lipids and a pH gradient across the membrane [6].

\section{Summary.}

We systematically analysed vesicle shapes of minimal bending energy for toroidal topology in two steps. First, we followed the established technique of solving the Euler Lagrange equations for axisymmetric shapes numerically as in the case of spherical topology. These axisymmetric solutions can be classified into sheets. We find sheets of discoid, sickle-shaped and, for the first time, up/down asymmetric toroidal stomatocytes, see figure 1. In a second step, we analysed the stability of these shapes with respect to conformal transformations which break the axisymmetry. Even though this cannot replace a full stability analysis, this method provides a lower bound on the extension of non-axisymmetric shapes within a phase diagram. However, it is even exact for a special shape, the Clifford torus, and should, by continuity, give a good approximation to the phase boundaries in a certain neighborhood of this shape.

The phase diagrams which are obtained by this two-step procedure have been determined for three different curvature models, the spontaneous-curvature (SC) model, the bilayer-couple (BC) model and the area-difference elasticity-model (ADE-model), see figures 4, 5 and 6 . The phase diagrams in the two simplified models ( $\mathrm{SC}$ and $\mathrm{BC}$ model) are two-dimensional and are both characterized by a large region of non-axisymmetic shapes. In the BC model, all phase transitions are continuous and all sheets of shapes show up as shapes of minimal energy somewhere in the phase diagram. In contrast, the phase diagram of the SC model contains a discontinuous transition and the toroidal stomatocytes do not represent states of lowest energy in this model.

For a comparison with experiments we discussed the more realistic ADE-model which takes into account that the bilayer consists of two compressible monolayers. Its phase diagram, figure 6 , which constitutes our main result, has been obtained from the phase diagram of the BC model by a generalized Legendre transformation. It contains a large region of non-axisymmetric shapes. All transitions but one are continuous. All observations of toroidal vesicles available so far are consistent with this phase diagram. While the observed genus-1 tori can be located explicity therein, the two types of genus-2 vesicles reported in experimental work can be closely 


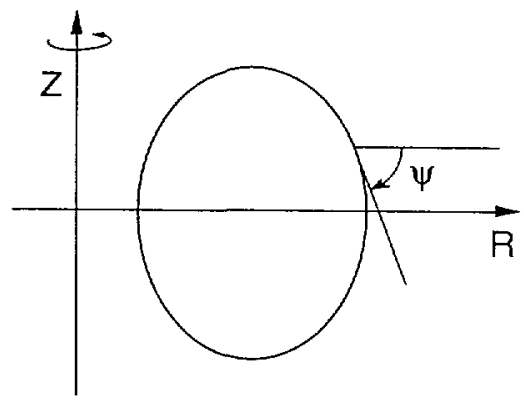

Fig.7. - Parametrization of an axisymmetric toroidal contour. $Z$ is the coordinate along the symmetry axis, $R$ is the distance from the axis and $\psi$ is the tilt angle of the contour.

related to genus-1 vesicles appearing in our phase diagram.

We then used this phase diagram for predictions of future experiments. Any axisymmetric torus should become non-axisymmetric when the temperature is decreased. A subsequent increase should render this shape axisymmetric again without any hysteresis effects since the axisymmetry breaking transition is predicted to be continuous. The predicted new type of axisymmetric shapes without reflection plane cannot easily be reached from known shapes by a change in temperature. One rather has to change the relative amount of lipid molecules in the two monolayers at fixed reduced volume.

In conclusion, we gave a fairly detailed analysis of toroidal shapes which minimize curvature energies. The phase diagram for a realistic curvature model provides the basis for a discussion of available experiments and should, as we hope, stimulate and guide the search for as yet unobserved but theoretically predicted shapes. For a quantitative comparison of theory and experiment, we also stress the relevance of systematic experiments where a parameter such as temperature is changed in a controlled fashion.

\section{Acknowledgments.}

We are grateful to X. Michalet, D. Bensimon and B. Fourcade for sending us video snapshots of genus-2 surfaces and reference [9] prior to publication.

\section{Appendix A.}

\section{Derivation of the shape equations for toroidal vesicles.}

Shapes with axial symmetry can be parametrized by three functions $R(S), Z(S)$ and $\psi(S)$, see figure 7. $R$ is the distance from the axis of symmetry, $Z$ the coordinate along the axis and $\psi$ the tilt angle of the contour, while $S$ is the arclength along the contour. The three functions $R, Z$ and $\psi$ are related by

$$
\dot{R}=\cos \psi \quad \text { and } \quad \dot{Z}=-\sin \psi
$$

The principal curvatures on the surface are $C_{1}=\dot{\psi}$ and $C_{2}=\sin \psi / R$.

The functional $F$ augmented with a term $\Sigma A+P V$ for the area and volume constraint has to be minimized with respect to $R, Z$ and $\psi$. Introducing two further Lagrange multiplier 
functions $\gamma(S)$ and $\eta(S)$ to ensure (A.1), the functional $F^{\prime}$ to be minimized can be written as

$$
F^{\prime} \equiv 2 \pi \kappa \int_{0}^{S_{1}} \mathrm{~d} S L
$$

Here,

$$
\begin{aligned}
L \equiv & \frac{R}{2}\left(\dot{\psi}+\frac{\sin \psi}{R}-C_{0}\right)^{2}+\bar{\Sigma} R+\frac{\bar{P}}{2} R^{2} \sin \psi+ \\
& \gamma(\dot{R}-\cos \psi)+\eta(\dot{Z}+\sin \psi)
\end{aligned}
$$

plays the role of a Lagrange function. The parameters $\bar{\Sigma}=\Sigma / \kappa$ and $\bar{P}=P / \kappa$ are the rescaled Lagrange multipliers for the volume and area constraints. From the condition $\delta F^{\prime}=0$, the Euler-Lagrange equations follow:

$$
\begin{aligned}
\dot{\psi} & =U \\
\dot{U} & =\frac{\cos \psi \sin \psi}{R^{2}}-\frac{U}{R} \cos \psi+\frac{\vec{P}}{2} R \cos \psi+\frac{\gamma}{R} \sin \psi+\frac{\eta}{R} \cos \psi \\
\dot{\gamma} & =\frac{1}{2}\left(U-C_{0}\right)^{2}-\frac{\sin ^{2} \psi}{2 R^{2}}+\bar{\Sigma}+\bar{P} R \sin \psi \\
\dot{\eta} & =0 \\
\dot{R} & =\cos \psi \\
\dot{Z} & =\sin \psi
\end{aligned}
$$

These are the shape equations for stationary shapes with axial symmetry. Note, that $\eta$ turns out to be constant while $\gamma=\gamma(S)$ is a function along the contour. The boundary conditions for $R, Z$ and $\psi$ are chosen to ensure a closed contour corresponding to a toroidal shape:

$$
\begin{aligned}
R(0) & =R\left(S_{1}\right)=R_{1} \\
Z(0) & =Z\left(S_{1}\right)=0 \\
\psi(0) & =-\pi / 2 \\
\psi\left(S_{1}\right) & =3 \pi / 2
\end{aligned}
$$

The boundary conditions for $\gamma$ and $U$ are

$$
\gamma\left(S_{1}\right)=\gamma(0) \text { and } U\left(S_{1}\right)=U(0)
$$

The variation of $F^{\prime}$ with respect to the total contour length $S_{1}$ leads to the condition $H\left(S_{1}\right)=0$, where

$$
\begin{aligned}
H \equiv & -L+\dot{\psi} \frac{\partial L}{\partial \dot{\psi}}+\dot{R} \frac{\partial L}{\partial \dot{R}}+\dot{Z} \frac{\partial L}{\partial \dot{Z}} \\
= & \frac{R}{2}\left(\dot{\psi}^{2}-\left(\frac{\sin \psi}{R}-C_{0}\right)^{2}\right)-\frac{\bar{P}}{2} R^{2} \sin \psi-\bar{\Sigma} R \\
& +\gamma \cos \psi-\eta \sin \psi
\end{aligned}
$$

is the Hamiltonian function which is conserved along the contour. Thus,

$$
\eta=-\frac{R_{1}}{2}\left(U(0)^{2}-\left(C_{0}+\frac{1}{R_{1}}\right)^{2}\right)-\frac{\bar{P}}{2} R_{1}^{2}+\bar{\Sigma} R_{1}
$$

is determined by the other boundary values to ensure $H\left(S_{1}\right)=0$. 


\section{Appendix B.}

\section{Stationary shapes and limit shapes.}

B.1 SheEts AND BRANCHES. - Stationary shapes of the energy functionals $F$ and $G$ can be parametrized by two independent parameters, e.g. $v$ and $c_{0}$, and, thus, form two-dimensional manifolds, or sheets [13]. Three different sheets of solutions of the shape equations (A4-A9) for toroidal vesicles can be distinguished: i) the sheet of discoid tori; ii) the sheet of sickle-shaped tori and iii) the sheet of toroidal stomatocytes. The classification and the topology of these sheets is unique and does not depend on a special model. Phase diagrams are determined by the energies $F$ and $G$ of the sheets as functions of $\left(v, c_{0}\right)$ and $(v, m)$, respectively. These functions are plotted in energy diagrams, which are cuts across the sheets along a one-dimensional line in the parameter space. Thus, the two-dimensional sheets are reduced to one-dimensional branches. One sheet can lead to more than one branch in an energy diagram, provided the sheet has one or several cusps. For a sheet with a cusp, two different cases can be distinguished: i) the cut cuts across the cusp, leading to an energy diagram which shows one branch with a cusp or ii) the cut does not cut across the cusp which leads to two separate branches in the energy diagram. Thus, the number of branches depends on i) how often the corresponding energy sheets are folded and ii) the precise location of the cut. As an example, figure 8 shows the energy $F$ as a function of $v$ for the cut $c_{0}=1$. This diagram exhibits four branches. Three of them can be uniquely related to three different sheets of stationary shapes. The fourth branch belongs to the sheet of discoid tori for $v<v_{\mathrm{cl}}$, and to the sheet of sickle-shaped tori for $v>v_{\mathrm{cl}}$. At $v=v_{\mathrm{cl}} \simeq 0.71$ this branch contains the Clifford torus CL where the two sheets are connected. Both sheets have wedges at $c_{0}<0$. This generates the fourth branch as a combination of two parts which meet at the Clifford torus. All shapes along this branch have an approximately circular cross section. Therefore, we call them circular tori.

B.2 Spontaneous-Curvature Model. - In the SC model only two sheets of stationary shapes are relevant for the phase diagram. These are: i) the sheet of discoid tori and ii) the sheet of sickle-shaped tori. The shapes on both sheets have a reflection symmetry plane. The sheets of toroidal stomatocytes bifurcate from both sheets and connect them, but for $-2<c_{0}<2$, which is the range we investigated, their energy $F$ is always larger than the energy of the shapes with reflection symmetry.

First, consider the energy $F$ for fixed spontaneous curvature $c_{0}$. As an example, figures $8 \mathrm{a}$, b show the energy $F$ of four branches as a function of $v$ for fixed $c_{0}=1$ : i) a branch of discoid tori; ii) a branch of sickle-shaped tori; iii) a branch of toroidal stomatocytes which bifurcates at the point $\mathrm{C}_{\text {sick }}$ from the branch of sickle-shaped tori and iv) a branch of circular tori which contains at $v=v_{\mathrm{cl}} \simeq 0.71$ the Clifford torus CL. Shapes with lowest energy are sickle-shaped tori for $v<v_{\mathrm{D}} \simeq 0.41$ and circular tori for $v>v_{\mathrm{D}}$. At $v=v_{\mathrm{D}}$ where the two branches cross, a discontinuous shape transformation $\mathrm{D}_{\mathrm{ax}}$ occurs. The branches of toroidal stomatocytes and the discoid tori end up in limit shapes $\mathrm{L}_{\text {sto }}$ and $\mathrm{L}_{\text {disc }}$ where the hole diameter vanishes. A different limit occurs for sickle-shaped tori which selfintersect at $\mathrm{SI}_{\text {sick }}$ with $v \simeq 0.03$. At $\mathrm{C}^{*}$ with $v \simeq 0.68$, the circular shapes become unstable with respect to axisymmetry breaking conformal transformations.

The energy $F$ versus $c_{0}$ is plotted for fixed $v=0.55$ in figures $9 \mathrm{a}, \mathrm{b}$. Three different branches occur for this cut. Figure 9 a shows the two branches with up-down reflection symmetry while figure $9 \mathrm{~b}$ shows the branches of toroidal stomatocytes only. The discoid branch exhibits a Gibbs loop which separates discoid tori from circular shapes on this branch. The sickle-shaped branch crosses the discoid branch for $c_{0} \simeq 0.1$ at the point $D_{\text {ax. }}$. At $C_{\text {sick }}^{(1)}, C_{\text {sick }}^{(2)}$ and $C_{\text {disc }}$, branches 

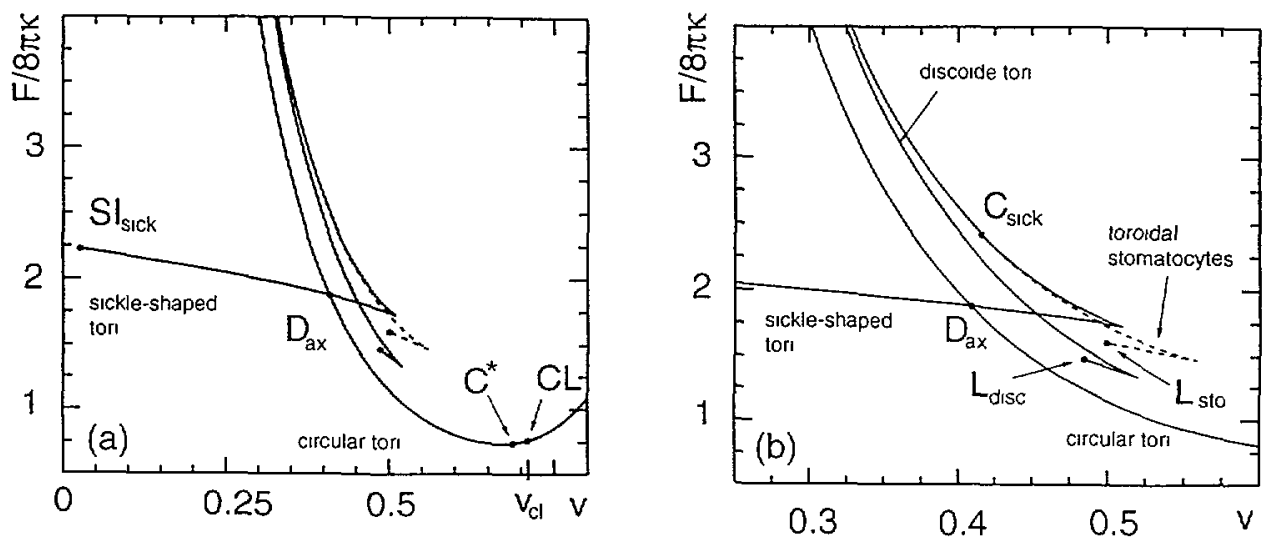

Fig.8. - Energy $F$ versus $v$ for $c_{0}=1.0:$ a) shows the complete branches and b) gives some details near $v \simeq 0.5$. Four branches are displayed. Three of them correspond to the different sheets of stationary shapes: one branch of discoid tori, one branch of sickle-shaped tori and one branch of toroidal stomatocytes. The branch of circular tori is for $v<v_{\mathrm{cl}} \simeq 0.71$ part of the discoid sheet and for $v>v_{\mathrm{cl}}$ part of the sickle-shaped sheet. For $v=v_{\mathrm{cl}}$ it contains the Clifford torus (CL) which connects the two sheets. At the point $\mathrm{C}_{\text {sick }}$, the toroidal stomatocytes bifurcate from the sickle-shaped branch. This branch ends up in a limit shape $L_{\text {sto }}$ with vanishing hole diameter. A similar limit shape $L_{\text {disc }}$ also exists for the branch of discoid tori.
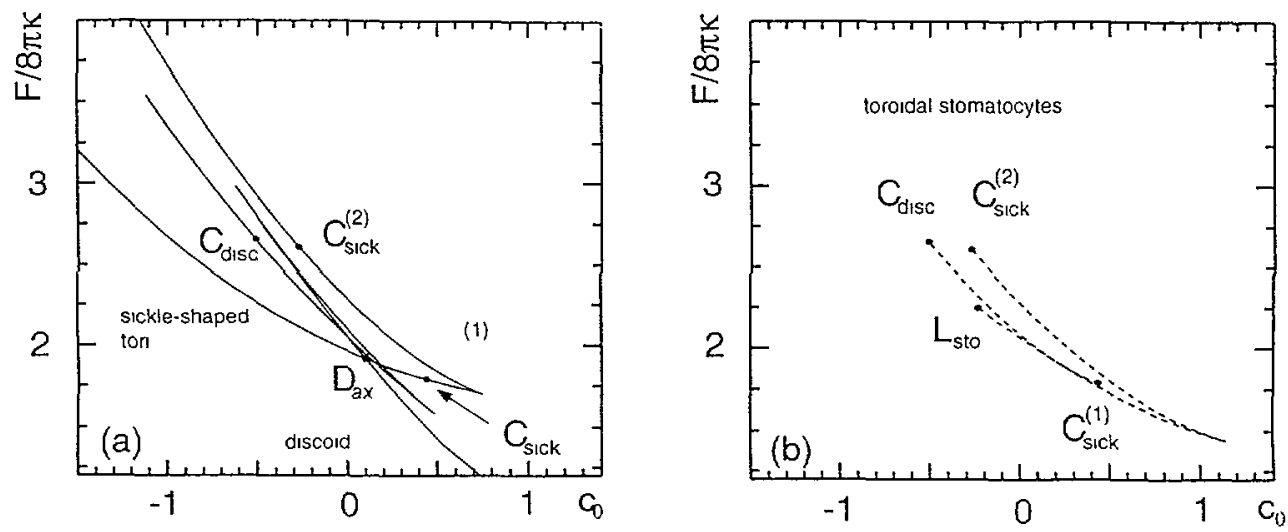

Fig.9. - Energy $F$ versus $c_{0}$ for $v=0.55$. In (a) the branches of discoid tori and sickle-shaped tori with reflection symmetry are displayed. The branches of toroidal stomatocytes are plotted in (b). The toroidal stomatocytes bifurcate from the other branches in the points $\mathrm{C}_{\text {sick }}^{(1)}, \mathrm{C}_{\text {sick }}^{(2)}$ and $\mathrm{C}_{\text {disc }}$. $\mathrm{L}_{\text {sto }}$ is a limit shape of these stomatocytes. The sickle-shaped branch and the branch of discoide tori cross in the point $D_{a x}$. In the phase diagram, this leads to a discontinuous shape tranformation from sickle-shaped tori to circular tori.

of toroidal stomatocytes bifurcate from the branches of sickle-shaped tori and discoid tori, respectively, and connect them. One branch of toroidal stomatocytes ends up in a limit shape $\mathrm{L}_{\text {sto }}$ with infinitesimal hole diameter. For $v>v_{\mathrm{cl}} \simeq 0.71$, the topology of the branches changes. In figure 10 , we show $F$ versus $c_{0}$ for $v=0.73$. In the $c_{0}$-interval chosen, only the sickle-shaped branch exists. This branch exhibits a Gibbs loop corresponding to the discontinuous transition 

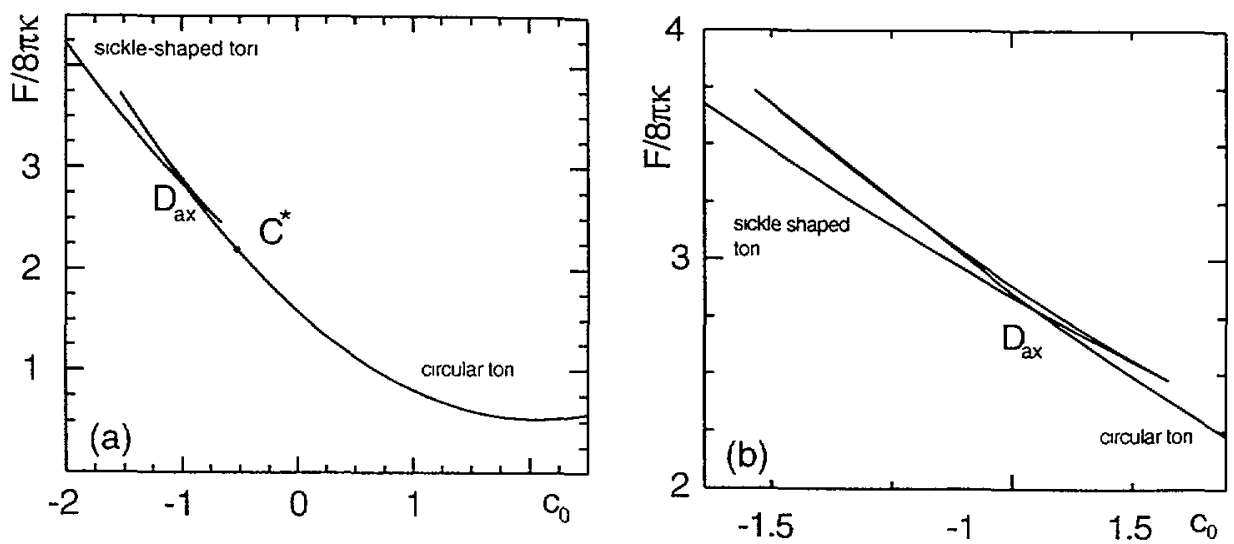

Fig.10. - Energy $F$ versus $c_{0}$ for $v=0.73$. The circular tori on the right hand side of $\mathrm{C}^{*}$ are unstable with respect to axisymmetry breaking. The Gibbs loop at $D_{a x}$ is enlarged in (b).

$D_{\mathrm{ax}}$ from sickle-shaped tori to shapes with a circular contour. These circular shapes become unstable with respect to axisymmetry breaking at $\mathrm{C}^{*}$ with $c_{0} \simeq-0.5$. Using these and further energy diagrams, we obtained the phase diagram for the SC model as shown in figure 4.

B.3 BilayeR-COUPLE MOdel. - In the BC model, the energy $G$ as a function of $v$ and $m$ determines the phase diagram. Figure 1a shows $G$ versus $m$ for the branches of stationary shapes with $v=0.55$. Three different branches, corresponding to the three sheets, are displayed. The branch of sickle-shaped tori starts for small $m$ at a limit shape $\mathrm{L}_{\text {sick. }}$. However, these shapes are unstable with respect to symmetry breaking conformal transformations. They become stable at the point $\mathrm{C}^{*}$ with $v \simeq 0.45$. Beyond the point $\mathrm{C}_{\text {sick }}^{(1)}$ where the branch of toroidal stomatocytes bifurcates from the branch of sickle-shaped tori, the branch of sickle-shaped tori corresponds to saddle points, i.e. it is locally unstable. For larger values of $m$, there is another bifurcation $\mathrm{C}_{\text {sick }}^{(2)}$ where a branch of toroidal stomatocytes meets the sickle-shaped branch. The branch of discoid tori starts from a limit shape $\mathrm{L}_{\text {circ }}$ with perfectly circular cross section. The point $\mathrm{C}^{*}$ close to $\mathrm{L}_{\text {circ }}$ indicates that $\mathrm{L}_{\text {circ }}$ is unstable with respect to perturbations which break the axisymmetry. The toroidal stomatocytes bifurcate from the discoide branch at $\mathrm{C}_{\text {disc }}$. The discoid branch ends up at a second limit shape $L_{\text {disc }}$ which is identical to a selfintersecting spherical oblate. Likewise, the toroidal stomatocytes reach a limit shape $\mathrm{L}_{\text {sto }}$ with infinitesimal hole. The energy diagram therefore leads to a sequence of shapes of minimal energy as shown in figure $1 \mathrm{~b}$. This scenario holds for $0.55<v<v_{\mathrm{cl}} \simeq 0.71$. For $v=v_{\mathrm{cl}}$ the branches of sickle-shaped and discoid tori meet in a cusp at the Clifford torus where the derivative $\partial G / \partial m$ is singular. For $v>v_{\mathrm{cl}}$, the energy of the discoid branch is larger than the energy of the sickle-shaped branch and the toroidal stomatocytes disappear. Figures 11a, b show the two branches for $v=0.73$. Here, the sickle-shaped branch begins at a limit shape $\mathrm{L}_{\text {circ }}$. Two points $\mathrm{C}^{*}$ of conformal instability occur on this branch. Between these points, axisymmetric shapes are stable.

Again, the information extracted from these and similar energy diagrams lead to the phase diagram for the $\mathrm{BC}$ model as shown in figure 5 . 

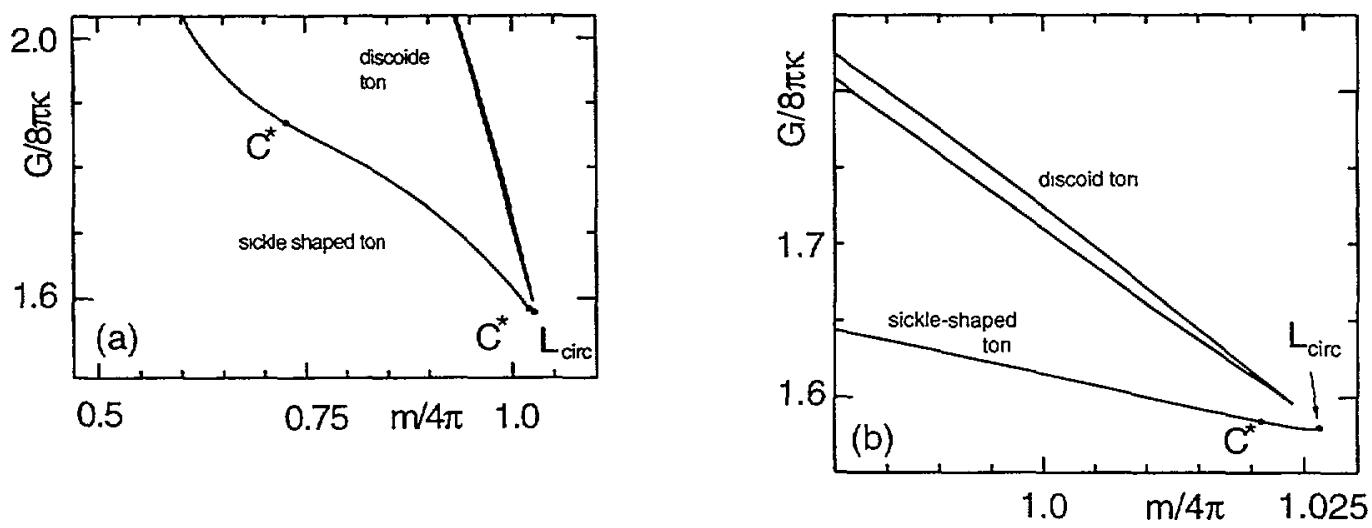

Fig.11. - Energy $G$ as a function of $m$ for fixed $v=0.73$. In this case, the sickle-shaped branch has always lowest energy. At $L_{\text {circ }}$, it ends up in a limit shape with exactly circular cross section. $C^{*}$ denotes the points of conformal instability.

B.4 Limit Shapes AND NECK CONDITION. - In this section, we discuss limit shapes, which are singularities on the sheets of stationary shapes. Different types of limit shapes occur for toroidal topology.

In the limit of large $c_{0}$ limit shapes $\mathrm{L}_{\text {circ }}$ with exactly circular cross section appear. In the $(v, m)$ plane these shapes fall on the hyperbola $m=3 \pi / v$.

Another type of limit shapes are tori with a hole of vanishing diameter, $R_{1}=0$. They are denoted: i) $L_{\text {disc }}$ on the branch of discoid tori; ii) $L_{\text {sick }}$ on the branch of sickle-shaped tori and iii) $\mathrm{L}_{\text {sto }}$ on the branch of toroidal stomatocytes, respectively, see figure 1 .

Toroidal shapes with an infinitesimal neck should be closely related to shapes of spherical topology which start to selfintersect. The numerical analysis shows, in fact, that the shapes with reflection plane $L_{\text {disc }}$ and $L_{\text {sick }}$ bifurcate from branches of selfintersecting shapes of spherical topology. The limit shapes $\mathrm{L}_{\text {disc }}$ form exactly the line of selfintersecting spherical oblates, denoted $\mathrm{SI}_{\mathrm{ob}}$ in reference [12]. At the point of selfintersection, an infinitesimal hole does not change the bending energy and, thus, the toroidal shape of minimal energy is identical to the spherical shape with the same $v$ and $m$.

The shapes $\mathrm{L}_{\text {sick }}$ can also be understood in terms of spherical shapes as follows. A spherical prolate $A$ is located within a perfect sphere $B$ touching the prolate at two points where the shapes are connected by infinitesimal necks. Both shapes have the same tension $\Sigma^{(A)}=\Sigma^{(B)}$ but opposite pressures $P^{(\mathrm{A})}=-P^{(\mathrm{B})}$ and spontaneous curvatures $C_{0}^{(\mathrm{A})}=-C_{0}^{(\mathrm{B})}$ These conditions are necessary to get a stationary toroidal combination of the two shapes of spherical topology. The sphere and the prolate can be connected by an infinitesimal neck provided the distance between the two poles of the prolate is equal to the diameter of the enclosed sphere. All these conditions can indeed be fulfilled simultaneously since for any fixed $P$ and $C_{0}$ ( $\Sigma$ is used to choose a length scale) there exists a spherical prolate and a sphere which coexist. One of the two parameters, $P$ and $C_{0}$, can now be used to change the sphere diameter in such a way that the prolate fits into the sphere. The second parameter is still free and therefore a whole line of limit shapes $\mathrm{L}_{\text {sick }}$ exists [35].

The limit shapes of vesicles of spherical topology which consist of one or more spherical shapes, have been found to be connected by ideal necks [12, 13]. Ideal necks of such spherical 


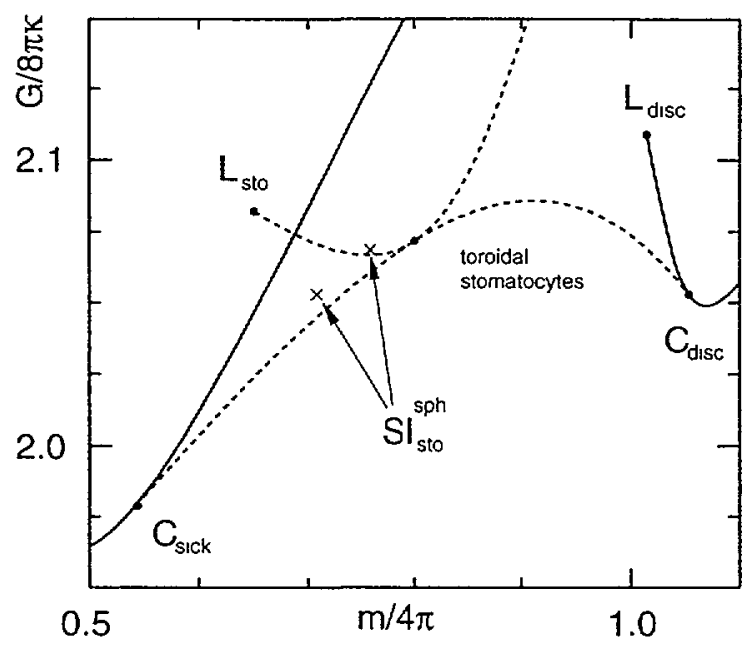

Fig.12. - Energy $G$ versus $m$ for $v=0.55$ which represents a part of figure 1. There are two branches of toroidal stomatocytes which seem to intersect with a common tangent. The lower branch connects the branch of sickle-shaped tori with the branch of discoid tori. The energies of shapes of spherical stomatocytes which selfintersect $\mathrm{SI}_{\text {sto }}^{\mathrm{sph}}$, are shown for comparison. They have a larger energy $G$ than toroidal stomatocytes.

shapes fulfill the neck condition

$$
C^{\mathrm{A}}+C^{\mathrm{B}}=C_{0}
$$

where $C^{\mathrm{A}}$ and $C^{\mathrm{B}}$ are the values of the curvatures of the two shapes adjacent to the neck $[12,13]$. The neck condition, thus, selects sets of spherical shapes which remain stationary shapes when connected by infinitesimal necks.

For the limit shapes $\mathrm{L}_{\text {disc }}$ and $\mathrm{L}_{\text {sick }}$ the numerical findings that these shapes occur along one-dimensional curves in the space of shapes shows that a neck condition similar to (B1) does not apply here. For any further condition would select only a discrete number of shapes along these curves.

Therefore, we conclude that the ideal neck condition (B1) is special to vesiculated shapes but does not apply to infinitesimal necks under all circumstances. Obviously, more work would be needed to understand this phenomenon mathematically.

Toroidal stomatocytes with vanishing hole diameter $\mathrm{L}_{\text {sto }}$ can not easily be reduced to spherical shapes. Even though they look very similar to spherical stomatocytes that begin to selfintersect $\mathrm{SI}_{\text {sto }}^{\mathrm{sph}}$, denoted $\mathrm{SI}_{\text {sto }}$ in reference [12], the numerical analysis reveals, that they are different. This is demonstrated in figure 12 where the branches of toroidal stomatocytes and the shapes $\mathrm{SI}_{\text {sto }}^{\mathrm{sph}}$ are shown to be separated. Thus, there is no obvious connection between the branches of spherical and toroidal stomatocytes and any further analysis of the shapes $\mathrm{L}_{\text {sto }}$ is difficult. In particular, their location in the phase diagram is hard to obtain accurately.

B.5 Relation to TwO-Dimensional vesicles. - For diverging hole diameter $R_{1}$ with fixed contour length $S_{1}=2 \pi R_{2}$, an axisymmetric torus reaches the limit of a long cylinder with reduced volume $v \sim 1 / R_{1}$ and area $A \approx 4 \pi^{2} R_{1} R_{2}$. This limit occurs for the unstable branches of both, the discoid shapes and the sickle-shaped tori, where for infinitesimal $v$ the 
energies $F$ and $G$ diverge. In this limit, the shape equations reduce to those of two-dimensional vesicles.

With the parametrization $R(S)=R_{1}+R_{2} r(S)$ of the contour, the functional $F^{\prime}$ as in (A2) expanded in lowest order in $R_{2} / R_{1}$ reads

$$
F^{\prime}=2 \pi \kappa \frac{R_{1}}{R_{2}}\left[\frac{1}{2} \int_{0}^{2 \pi} \mathrm{d} s\left(\dot{\psi}-C_{0} R_{2}\right)^{2}+2 \pi \bar{\Sigma} R_{2}^{2}+\bar{P} R_{2}^{3} \frac{A_{2 \mathrm{~d}}}{R_{2}^{2}}+O\left(R_{2} / R_{1}\right)\right]
$$

with the dimensionless integration variable $s=S / R_{2} . A_{2 \mathrm{~d}}$ denotes the area of the crosssection which is the area of a two-dimensional vesicle. Variation of (B2) for fixed $R_{1}$ and $R_{2}$ with respect to the shape leads to the shape equations of two-dimensional vesicles [36] which describe stationary solutions of the functional

$$
f_{2 \mathrm{~d}}^{\prime} \equiv f_{2 \mathrm{~d}}+p_{2 \mathrm{~d}} \frac{A_{2 \mathrm{~d}}}{R_{2}^{2}}
$$

with

$$
f_{2 \mathrm{~d}} \equiv \frac{1}{2} \int_{0}^{2 \pi} \mathrm{d} s \dot{\psi}^{2}
$$

The minimum of $F^{\prime}$ can then be expressed by the minimal energy $f_{2 \mathrm{~d}}^{\prime}\left(p_{2 \mathrm{~d}}\right)$ with $p_{2 \mathrm{~d}}=\vec{P} R_{2}^{3}$ as

$$
F^{\prime}=2 \pi \kappa \frac{R_{1}}{R_{2}}\left(f_{2 \mathrm{~d}}^{\prime}\left(\bar{P} R_{2}^{3}\right)-2 \pi C_{0} R_{2}+\pi C_{0}^{2} R_{2}^{2}+2 \pi \bar{\Sigma} R_{2}^{2}+O\left(R_{2} / R_{1}\right)\right)
$$

Variation of (B5) with respect to $R_{1}$ leads to

$$
f_{2 \mathrm{~d}}^{\prime}\left(\bar{P} R_{2}^{3}\right)-2 \pi C_{0} R_{2}+\pi C_{0}^{2} R_{2}^{2}+2 \pi \bar{\Sigma} R_{2}^{2}=0
$$

A second condition results from varying (B5) with respect to $R_{2}$ :

$$
3 \bar{P} R_{2}^{3} \frac{\partial f_{2 \mathrm{~d}}^{\prime}}{\partial p_{2 \mathrm{~d}}}\left(\bar{P} R_{2}^{3}\right)-2 \pi C_{0} R_{2}+2 \pi R_{2}^{2}\left(C_{0}^{2}+2 \bar{\Sigma}\right)=0
$$

Using $\partial f_{2 \mathrm{~d}}^{\prime} / \partial p_{2 \mathrm{~d}}=A_{2 \mathrm{~d}} / R_{2}^{2}$ and combining (B6) and (B7) leads to an additional condition on a two-dimensional vesicle contour being a solution in the limit of infinitesimal $v$ for toroiodal topology:

$$
f_{2 \mathrm{~d}}^{\prime}-\frac{3}{2} p_{2 \mathrm{~d}} \frac{A_{2 \mathrm{~d}}}{R_{2}^{2}}=\pi C_{0} R_{2}
$$

With equation (B3), this can be rewritten as

$$
f_{2 \mathrm{~d}}=\frac{1}{2} p_{2 \mathrm{~d}} \frac{A_{2 \mathrm{~d}}}{R_{2}^{2}}+\pi C_{0} R_{2}
$$

The limit of large $R_{1}$ with $R_{2}$ fixed is now taken for fixed reduced spontaneous curvature $c_{0}$, which implies that $C_{0}$ is rescaled according to $C_{0} \approx c_{0} /\left(R_{1} R_{2}\right)^{1 / 2}$ Since $C_{0}$ becomes zero for diverging $R_{1}$, equation (B9) has to be solved for $C_{0}=0$. Solutions can be found by plotting the energy $f_{2 \mathrm{~d}}$ of two-dimensional stationary shapes together with $\frac{1}{2} p_{2 \mathrm{~d}} A_{2 \mathrm{~d}} / R_{2}^{2}$ versus $p_{2 \mathrm{~d}}$ [36]. Two solutions exist: i) a circular contour which fulfills the cylinder equations for threedimensional vesicles [12], and ii) an ellipsoidal contour with $p_{2 \mathrm{~d}}=p^{*}$ which is part of the line of non-circular shapes of two-dimensional vesicles. This two-dimensional ellipsoidal contour taken as a cross-section of a toroidal vesicle with the long axis parallel to the axis of symmetry corresponds to the limit shape of the unstable sickle-shaped branch for small $v$. Likewise, the same cross-section with the long axis perpendicular to the axis of symmetry corresponds to the limit shape of discoid tori. Finally, the circular cross-section corresponds to the limit of circular tori for small $v$. 


\section{Appendix C.}

\section{Stability with respect to special conformal transformations.}

We restrict the stability analysis to axisymmetric shapes with an additional reflection plane. The origin of the coordinate system is chosen where the symmetry axis crosses the reflection plane. Under a special conformal transformation, parametrized by the vector a, the transformed area $A$ expanded to second order in $\mathrm{a}$ is

$$
A^{\prime}=A-2 \mathbf{a}^{2} \oint \mathrm{d} A R^{2}+12 \oint \mathrm{d} A(\mathbf{a} \cdot \mathbf{R})^{2}+\mathbf{O}\left(\mathbf{a}^{4}\right)
$$

The special conformal transformation is followed by a scale transformation $\mathbf{R}^{\prime \prime}=\lambda \mathbf{R}$ with $\lambda=\left(A^{\prime} / A\right)^{1 / 2}$ which restores the surface area $A$. The transformed volume $V$ and total mean curvature $M$ expanded in a read

$$
V^{\prime \prime}=\lambda^{3} V^{\prime}=V\left(1+a_{\alpha} B_{\alpha \beta}^{(V)} a_{\beta}\right)+O\left(a^{4}\right)
$$

and

$$
M^{\prime \prime}=\lambda M^{\prime}=M\left(1+a_{\alpha} B_{\alpha \beta}^{(M)} a_{\beta}\right)+O\left(a^{4}\right)
$$

For axisymmetric shapes, $B_{\alpha \beta}^{(V)}$ and $B_{\alpha \beta}^{(M)}$ can be expressed by integrals along the contour functions $R(S), Z(S)$ and $\psi(S)$. The stability analysis with respect to modes with $a_{Z}=0$, which break the axisymmetry, require the calculation of

$$
B_{X X}^{(V)}=B_{Y Y}^{(V)}=\frac{12 \pi}{A} \int_{0}^{S_{1}} \mathrm{~d} S\left(R Z^{2}-2 R^{3}\right)+\frac{4 \pi}{V} \int_{0}^{S_{1}} \mathrm{~d} S\left(9 R^{3} Z-R Z^{3}\right) \cos \psi
$$

and

$$
\begin{aligned}
B_{X X}^{(M)}= & B_{Y Y}^{(M)}=-\frac{6 V}{M}+\frac{2 \pi}{M} \int_{0}^{S_{1}} \mathrm{~d} S\left(R^{3}-R Z^{2}\right)\left(U+\frac{\sin \psi}{R}\right) \\
& +\frac{16 \pi}{M} \int_{0}^{S_{1}} \mathrm{~d} S R^{2} \sin \psi+\frac{4 \pi}{A} \int_{0}^{S_{1}} \mathrm{~d} S\left(R Z^{2}-2 R^{3}\right)
\end{aligned}
$$

For a derivation of the stability criterion, first consider the SC model. Choose a branch of stationary shapes for fixed $C_{0}$ and $A$ but varying volume $V$. Let $F(V)$ denote the energy of these shapes. Choose a stationary shape $\mathcal{S}_{1}$ along this branch with volume $V_{1}$, energy $F\left(V_{1}\right)$ and total mean curvature $M_{1}$. A special conformal transformation with subsequent rescaling applied to this shape $\mathcal{S}_{1}$, generates a non-axisymmetric shape $\mathcal{S}_{1}^{\prime \prime}$ with energy

$$
F_{\mathrm{SCT}}(\mathbf{a})=F\left(V_{1}\right)-2 \kappa C_{0}\left(M_{1}^{\prime \prime}(\mathbf{a})-M_{1}\right),
$$

volume $V_{1}^{\prime \prime}(\mathbf{a})$ and total mean curvature $M_{1}^{\prime \prime}(\mathbf{a})$.

To lowest order in $V_{1}^{\prime \prime}-V_{1}$ and $M_{1}^{\prime \prime}-M_{1}$, the energy $F_{\mathrm{SCT}}$ defined in equation (C6) changes linearly as a function of $V_{1}^{\prime \prime}$ :

$$
F_{\mathrm{SCT}}\left(V_{1}^{\prime \prime}\right)=F\left(V_{1}\right)-2 \kappa C_{0} \frac{M_{1} B_{X X}^{(M)}}{V_{1} B_{X X}^{(V)}}\left(V_{1}^{\prime \prime}-V_{1}\right)+O\left(\left(V_{1}^{\prime \prime}-V_{1}\right)^{2}\right)
$$

On the other hand, along the branch $F(V)$, there exists a shape $\mathcal{S}^{\text {axi }}$ with volume $V_{1}^{\prime \prime}$ which has an energy $F\left(V_{1}^{\prime \prime}\right)$, compare figure 13. This energy $F\left(V_{1}^{\prime \prime}\right)$ expanded to lowest order in 


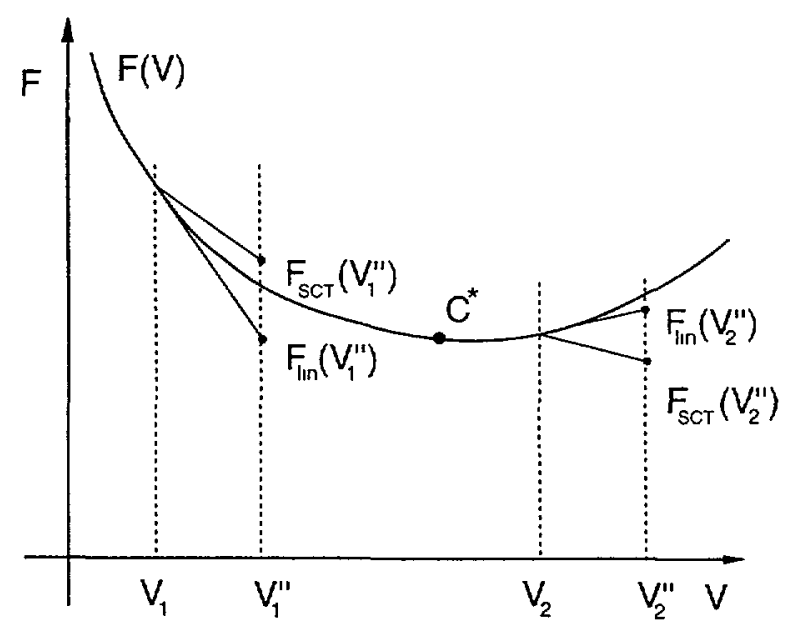

Fig.13. - Stability analysis with respect to special conformal transformations for shapes on an axisymmetric branch of stationary shapes with energy $F(V)$. Starting with a shape $\mathcal{S}_{1}$ with volume $V_{1}$ and energy $F\left(V_{1}\right)$, an infinitesimal special conformal transformation generates a new shape $\mathcal{S}_{1}^{\prime \prime}$ with volume $V_{1}^{\prime \prime}$ and energy $F_{\mathrm{SCT}}\left(V_{1}^{\prime \prime}\right)$. This energy has to be compared to $F_{\operatorname{lin}}\left(V_{1}^{\prime \prime}\right)$ of a shape $\mathcal{S}^{\text {axi }}$ on the branch. The figure shows two cases: i) on the left hand side of $\mathrm{C}^{*}, F_{\mathrm{SCT}}\left(V_{1}^{\prime \prime}\right)>F_{\text {lin }}\left(V_{1}^{\prime \prime}\right)$ shows that the branch is locally stable; ii) on the right hand side of $\mathrm{C}^{*} F_{\mathrm{SCT}}\left(V_{2}^{\prime \prime}\right)<F_{\text {lin }}\left(V_{2}^{\prime \prime}\right)$ indicates, that the branch is unstable with respect to special conformal transformations.

$V_{1}^{\prime \prime}-V_{1}$ is given by

$$
F_{\text {lin }}\left(V_{1}^{\prime \prime}\right)=F\left(V_{1}\right)+\frac{\partial F}{\partial V}\left(V_{1}^{\prime \prime}-V_{1}\right)+O\left(\left(V_{1}^{\prime \prime}-V_{1}\right)^{2}\right)
$$

If the slope of $F_{\mathrm{SCT}}\left(V_{1}^{\prime \prime}\right)$ is smaller than the slope of $F\left(V_{1}^{\prime \prime}\right)$, i.e. if $F\left(V_{1}^{\prime \prime}(\mathrm{a})\right) \leq F_{\mathrm{SCT}}(\mathrm{a})$ in the limit of small a, a special conformal transformation will generate a non-axisymmetric shape $\mathcal{S}_{1}^{\prime \prime}$ with volume $V_{1}^{\prime \prime}$ which has a lower energy than the stationary shape $\mathcal{S}^{\text {axi }}$ with the same volume. The axisymmetric shape $\mathcal{S}^{\text {axi }}$ with volume $V_{1}^{\prime \prime}$ is therefore not a shape of lowest energy. The perturbation is marginal when both slopes are equal. The stability criterion obtained by these arguments for the SC model thus reads [30]

$$
\frac{\partial F}{\partial V} V B_{X X}^{(V)}+2 \kappa C_{0} M B_{X X}^{(M)} \leq 0
$$

Note, that $\partial F / \partial V=-\kappa \bar{P}$, where $\bar{P}$ is known from solving the shape equations.

For the $\mathrm{BC}$ model, the energy $G(V, M)$ of a branch of axisymmetric shapes is invariant under a special conformal transformation. A non-axisymmetric shape $\mathcal{S}_{1}^{\prime \prime}$, generated from a shape $\mathcal{S}_{1}$ with energy $G\left(V_{1}, M_{1}\right)$ by a special conformal transformation has the energy $G_{\mathrm{SCT}}(\mathrm{a})=$ $G\left(V_{1}, M_{1}\right)$, volume $V_{1}^{\prime \prime}(\mathrm{a})$ and total mean curvature $M_{1}^{\prime \prime}(\mathrm{a})$. Comparison of the energy of shape $\mathcal{S}_{1}^{\prime \prime}$ with the energy $G\left(V_{1}^{\prime \prime}, M_{1}^{\prime \prime}\right)$ of a shape $\mathcal{S}^{\text {axi }}$ along the branch of axisymmetric stationary shapes leads to the stability criterion

$$
G_{\mathrm{SCT}}\left(V_{1}^{\prime \prime}(\mathrm{a}), M_{1}^{\prime \prime}(\mathrm{a})\right) \leq G\left(V_{1}, M_{1}\right)
$$


Expanding

$$
\begin{aligned}
G\left(V_{1}^{\prime \prime}, M_{1}^{\prime \prime}\right)= & G\left(V_{1}, M_{1}\right)+\frac{\partial G}{\partial V}\left(V_{1}-V_{1}^{\prime \prime}\right)+\frac{\partial G}{\partial M}\left(M_{1}-M_{1}^{\prime \prime}\right) \\
& +O\left(\left(V_{1}-V_{1}^{\prime \prime}\right)^{2}\right)+O\left(\left(M_{1}-M_{1}^{\prime \prime}\right)^{2}\right)
\end{aligned}
$$

together with

$$
\frac{\partial G}{\partial M}=2 \kappa C_{0}
$$

again leads to the criterion (C9) as it was obtained for the $\mathrm{SC}$ model.

For shapes without reflection plane, generalized stability criteria can be obtained which differ for the BC model and the SC model. We have not checked the toroidal stomatocytes for their conformal stability. However, we believe that they are conformally stable in all regions of the phase diagram where they have lower energy than the shapes with a reflection plane for the following reason. We find that the axisymmetric shapes from which the toroidal stomatocytes bifurcate are conformally stable. By continuity, this will hold at least in the vicinity of the bifurcation for the toroidal stomatocytes too.

\section{References}

[1] Lipowsky R., Nature 349 (1991) 475.

[2] Wortis M., Seifert U., Berndl K., Fourcade B., Miao L., Rao M., Zia R.K.P., Proceedings of the workshop on Dynamical Phenomena at Interfaces, Surfaces and Membranes, D. Beysens, N. Boccara, G. Forgacs Eds. (Nova Science).

[3] The Structure and Conformation of Amphiphilic Membranes, R. Lipowsky, D. Richter, K. Kremer Eds. (Springer, Berlin, 1992).

[4] Berndl K., Käs J., Lipowsky R., Sackmann E., Seifert U., Europhys. Lett. 13 (1990) 659.

[5] Käs J., Sackmann E., Biophys. J. 60 (1991) 825.

[6] Fargé E., Devaux P.F., Biophys. J. 61 (1992) 347.

[7] Mutz M., Bensimon D., Phys. Rev. A 43 (1991) 4525.

[8] Fourcade B., Mutz M., Bensimon D., Phys. Rev. Lett. 68 (1992) 2551.

[9] Michalet X., Bensimon D., Fourcade B., Proceedings of the workshop on Soft order in physical systems (Les Houches, Feb. 16 - Feb. 25, 1993).

[10] Helfrich W., Z. Naturforsch., C28 (1973) 693.

[11] Evans E., Biophys. J. 14 (1974) 923.

[12] Seifert U., Berndl K., Lipowsky R., Phys. Rev. A 44 (1991) 1182.

[13] Miao L., Fourcade B., Rao M., Wortis M., Zia R.K.P., Phys. Rev. A 43 (1991) 6843.

[14] Svetina S., Žekš B., Eur. Biophys. J. 17 (1989) 101.

[15] Seifert U., Miao L., Döbereiner H.-G., Wortis M., p. 93 in reference [3];

Miao L., Ph.D. thesis, Simon Fraser University (1992);

Miao L., Seifert U., Wortis M., Döbereiner H.-G., submitted to Phys. Rev. E.

[16] Wiese W., Harbich W., Helfrich W., J. Phys. condensed matter 4 (1992) 1647.

[17] Evans E., Yeung A., Waugh R., Song J., p. 148 in reference [3].

[18] Waugh R.E., Song J., Svetina S., Žekš B., Biophys. J. 61 (1992) 974.

[19] Seifert U., J. Phys. Colloq. France 41 (1990) C7-339.

[20] Seifert U., Phys. Rev. Lett. 66 (1991) 2404. 
[21] Willmore T.J., Total Curvature in Riemannian Geometry (Ellis Horwood Ltd., Chichester, 1982).

[22] Duplantier B., Physica A 168 (1990) 179;

Duplantier B., Goldstein R.E., Romero-Rochin V., Pesci A.I., Phys. Rev. Lett. 65 (1990) 508.

[23] This corrects an erroneous statement in reference [20], where a two-fold degeneracy of the ground state was claimed even if the constraint on the area to volume ratio is taken into account.

[24] Mosseri R., Sadoc J.F., Charvolin J., p. 97 in reference [3].

[25] Fourcade B., J. Phys. II France 2 (1992) 1705.

[26] The difference between branches and sheets is discussed in appendix B.1.

[27] For the long axis of the ellipse perpendicular to the axis of symmetry, the tori become discoid, while for the long axis parallel to the axis of symmetry, the tori become sickle-shaped.

[28] Zhong-can Ou-Yang, Phys. Rev. A 41 (1990) 4517.

[29] In fact, for $v \approx 1$ toroidal shapes of lowest energy cannot be axisymmetric, since a axisymmetric shape with $v \approx 1$ has to look like a sphere with a hole along the axis of rotational symmetry. For vanishing hole diameter, the bending energy of such a shape diverges. Thus, the shape with lowest energy is a sphere with an infinitesimal handle, which is necessarily non-axisymmetric.

[30] Seifert U., J. Phys. A Math. Gen. 24 (1991) L573.

[31] Technically speaking, these shapes are for $v\left\langle v_{\mathrm{cl}}\right.$ part of the discoid sheet and for $v>v_{\mathrm{cl}}$ part of the sheet of sickle-shaped tori, see appendix B.1.

[32] In the phase diagram of the $\mathrm{SC}$ model, we have distinguished two lines $\mathrm{C}_{\mathrm{disc}}^{*}$ and $\mathrm{C}_{\text {sick }}^{*}$ of axisymmetry breaking. They originate from the same instability $\mathrm{C}^{*}$ but appear separated into two parts by the line of discontinuous transitions $D_{a x}$.

[33] In appendix $\mathrm{C}$, the criterion of conformal stability of axisymmetric shapes with a reflection plane is shown to be the same in the SC and the BC model. The generalization of this analysis for arbitrary values of $\alpha$ shows that the criterium is, in fact, independent of $\alpha$.

[34] Jülicher F., Seifert U., Lipowsky R., Phys. Rev. Lett. 71 (1993) 452.

[35] Another type of limit of sickle-shaped tori exists for $v \rightarrow 0$ and $c_{0} \leq 0$. These shapes consist of two identical spheres of radius $R_{0}$ which are connected by two infinitesimal necks. The two spheres can coexist in a toroidal shape if $\bar{P}=2 \mathrm{C}_{0} / R_{0}^{2}, \bar{\Sigma}=-\mathrm{C}_{0}^{2} / 2$. From these shapes, the line $\mathrm{L}_{\text {sick }}$ bifurcates at $c_{0} \simeq-4.24$. This bifurcation corresponds to the bifurcation of the prolate branch from the branch of spheres in the case of spherical shapes.

[36] Seifert U., Phys. Rev. A $\mathbf{4 3}$ (1991) 6803. 